\title{
Expression and the clinical significance of Wnt10a and Wnt10b in endometrial cancer are associated with the Wnt/ $\beta$-catenin pathway
}

\author{
HONGMAN CHEN $^{1,2}$, YINGMEI WANG ${ }^{1}$ and FENGXIA XUE ${ }^{1}$ \\ ${ }^{1}$ Department of Gynecology and Obstetrics, Tianjin Medical University General Hospital, Tianjin 300052; \\ ${ }^{2}$ Department of Gynecology and Obstetrics, The Fifth Central Hospital of Tianjin, Tianjin 300450, P.R. China
}

Received July 31, 2012; Accepted September 24, 2012

DOI: 10.3892/or.2012.2126

\begin{abstract}
To determine the role played by the Wnt/ $\beta$-catenin signaling pathway in the development of endometrial cancer (EC), we examined the expression of Wnt10a and Wnt10b in EC tissues and the correlation between their expression. Furthermore, the associations between these two proteins and the clinicopathological characteristics and prognosis of EC were also evaluated. In our search of alternative mechanisms, we investigated the impact of Wnt10b on proliferation and apoptosis of EC cells. Western blotting, 3-(4,5-dimethylthiazol2-yl)-2,5-diphenyltetrazolium bromide (MTT) assay and flow cytometry were used to evaluate the expression of Wnt10b and some key proteins of the $\mathrm{Wnt} / \beta$-catenin pathway, proliferation and apoptosis in EC. Our results showed that Wnt10b expression in EC tissues was significantly higher compared to that in hyperplastic and normal samples. The expression of Wnt10a in endometrioid cancer tissues was higher compared to that in other types of cancerous samples. The difference in Wnt10b levels was significant among subgroups for histological type, grade of differentiation, FIGO phase and lymphovascular metastasis. Furthermore, no correlation was observed between the expression of Wnt10a and Wnt10b. In the follow-up, Wnt10b gene expression was frequently upregulated in EC and associated with better prognostic clinicopathological markers in EC patients. Collectively, the in vitro data showed that the upregulated expression of Wnt10b in Ishikawa cells promoted proliferation and inhibited apoptosis through $\beta$-catenin and c-myc activation and adenomatous polyposis coli (APC) inhibition, which suggests that Wnt10b activates EC via the $W n t / \beta$-catenin pathway. These results suggest that Wnt10b likely plays an important role in the development of EC. Furthermore, these results identify a role for Wnt10b in EC cells through promoting proliferation and inhibiting apop-
\end{abstract}

Correspondence to: Dr Fengxia Xue, Department of Gynecology and Obstetrics, Tianjin Medical University General Hospital, 154 Anshan Road, Heping, Tianjin 300052, P.R. China

E-mail: fengxiaxue1962@163.com

Key words: endometrial cancer, Wnt10a, Wnt10b, proliferation, apoptosis, Wnt/ $\beta$-catenin pathway tosis, primarily through the activation of the Wnt/ $\beta$-catenin pathway. The role played by Wnt10a in EC, however, still requires further investigation.

\section{Introduction}

Endometrial cancer (EC) is one of the most common gynecologic malignancies in developed countries. In the US alone, 46,470 new cases and 8,120 deaths from EC were estimated in 2011 (1). In China, the incidence of this malignancy has also been observed to increase rapidly, threatening women's health (2). However, the etiology of this malignancy is not clearly understood. To date, the prevailing hypothesis is 'unopposed estrogen'. EC is commonly classified into two major types: type I estrogen-dependent and type II non-estrogen-dependent. The majority of EC cases are type I (approximately 80-90\%), which generally includes low-grade endometrioid histologies, often arising from a background of endometrial hyperplasia and may have more favorable prognoses (3). Althrough earlystage EC is often curable with surgery alone, with a 5-year survival rate of $75-93 \%$, the prognosis for late-stage disease is poor and the median survival for women with advanced or recurrent disease is approximately 1 year (4). Little is known concerning the molecular characteristics of EC that predict who will have recurrence and who should receive a particular type of treatment. Therefore, early diagnosis is vital, and identification of novel molecular biomarkers and therapeutic targets is imperative.

Wnt genes were first discovered by Nusse in 1982 (5). The Wnt family consists of at least 19 secreted-type glycoproteins with conserved 22-24 cysteine residues that play key roles in carcinogenesis and embryogenesis. Among these glycoproteins is the Wnt10a gene, which is located at human chromosome $2 \mathrm{q} 35$. The Wnt10b gene is located at human chromosome $12 \mathrm{q} 13$ (6). The Wnt10a protein binds to seven transmembrane-type Wnt receptors (FZD1-FZD10), while the Wnt10b protein has been shown to functionally interact with FZD5. Wnt10a has been shown to be most homologous to Wnt10b (59.2\% amino acid identity) (7); the two proteins are nearly identical, suggesting that duplication might have occurred during evolution, thus conserving these primordial clusters of genes during evolution. The Wnt signaling pathway regulates diverse developmental processes, such as cell migration, adhesion, proliferation and apoptosis. Previous studies 
have demonstrated that numerous malignant carcinomas, including osteosarcoma (8), gastrointestinal (9), prostate (10), breast (11) and ovarian cancer (12), are associated with an abnormal Wnt signaling pathway. The pathway is best known, however, for its role in colorectal cancer (CRC), in which greater than $90 \%$ of CRC cases carry an activated mutation in the Wnt signaling pathway, most frequently in the form of a mutational inactivation of adenomatous polyposis coli (APC) (13). Studies have reported that Wnt10a expression is upregulated in CRC cell lines (14) and Wnt10b expression is upregulated via the $\mathrm{Wnt} / \beta$-catenin pathway in breast cancer cell lines (15). Although previous reports have shown that $10-45 \%$ of all EC cases carry $\beta$-catenin mutations, with a slightly higher propensity in endometrioid EC (16), the role of Wnt signaling in EC has not been fully elucidated. The functional relationship and associated prognostic values between the Wnts and EC have not been determined. There is little information currently available concerning the relationship between clinicopathological characteristics and Wnts. Wang et al (17) reported the characterization of genomic alterations in five commonly used EC cell lines (HEC1A, HEC1B, AN3CA, ECC-1 and Ishikawa) and provided valuable genomic information for research focused on Wnt pathways in EC. The authors found that the Wnt10a gene was deleted in the HEC1B and AN3CA cell lines and was normal in the other three EC cell lines; the Wnt10b gene was amplified in the ECC-1 and Ishikawa cell lines and was normal in the other three EC cell lines.

In the present study, we investigated the expression of Wnt10a and Wnt10b in EC samples, and the relationship between expression levels and the clinicopathologic features of EC was also evaluated. Furthermore, the effect of the Wnt/ $\beta$ catenin pathway on the development of EC was investigated to further understand the underlying mechanism.

\section{Materials and methods}

Reagents and antibodies. Anti-Wnt10a rabbit anti-human and anti-Wnt10b mouse anti-human monoclonal antibodies were purchased from Abcam (St. Louis, MO, USA) (ab62051 and ab91201). Anti- $\beta$-catenin antibody was also purchased from Abcam. Anti-APC and anti-c-myc antibodies were purchased from Fisher Sigma. We used DMEM supplemented with $10 \%$ (vol/vol) fetal bovine serum (FBS) for the cell cultures. The 3-(4,5-dimethylthiazol-2-yl)-2,5-diphenyltetrazolium bromide (MTT) was obtained from Sigma (St. Louis, MO, USA). An Annexin V-fluorescein isothiocyanate (FITC) apoptosis detection kit was obtained from BD Biosciences (San Diego, CA, USA). Unless stated otherwise, all chemical reagents were purchased from Sigma.

Tissue specimens. All endometrium tissue specimens were obtained from patients who had undergone curettage or hysterectomy at the Tianjin Medical University, General Hospital between January 2001 and December 2010. None of the patients had accepted any radiation, chemotherapy or hormonal therapy prior to surgery. These specimens included 84 normal endometrium (48 in the proliferative phase and 36 in the secretory phase), 54 endometrial hyperplasias (18 simple, 6 complex and 30 atypical hyperplasias) and 102 endometrial carcinomas (83 endometrioid, 12 adenosquamous, 4 uterine mucus, 2 uterine papillary serous and 1 clear cell carcinoma). The median age of the patients with normal endometrium, endometrial hyperplasia and carcinoma were 51, 49 and 52 years, respectively. Of the 102 EC patients, 75 were diagnosed as stage I, 5 as stage II and 22 as stage III, according to FIGO 2009 staging. Our study was approved by the local ethics committees of Tianjin Medical University, General Hospital. All subjects provided written consent to participate in our investigation. Two gynecological pathologists reviewed the tumor slides to confirm the original diagnoses. These tissue specimens were constructed into tissue chips.

Immunohistochemistry staining and scoring. The specimens were fixed in a $10 \%$ formalin solution and embedded by routine methods in paraffin for sectioning at a thickness of $3 \mu \mathrm{m}$. Immunohistochemical analysis was performed using the streptavidin-biotin amplification method with a Histofine kit. Sections were deparaffinized and incubated for $30 \mathrm{~min}$ with $3 \% \mathrm{H}_{2} \mathrm{O}_{2}$ in methanol to block endogenous peroxidase activity. After being rinsed in Tris-buffered saline, the sections were incubated for $6 \mathrm{~h}$ at room temperature with a monoclonal antibody directed against anti-Wnt10a rabbit anti-human (1:100 dilution) and anti-Wnt10b mouse anti-human monoclonal antibody (1:200 dilution). The antibody complex was visualized with 3,3'-diaminobenzidine tetrahydrochloride (DAB) solutions. Pancreatic cancer and breast cancer tissues were used as positive controls. For the negative control, phosphate-buffered saline was substituted for the primary antibody.

Two observers blindly and independently assessed the immunohistochemical expression of Wnt10a and Wnt10b. Positive immunostaining for Wnt10a and Wnt10b was detected both on the membrane of the cells and in the cytoplasm. To evaluate Wnt10a and Wnt10b, we defined a score that corresponded with the sum of the percentage of positive cells (0, $0-24 \%$ immunopositive cells; $1,25-50 \%$ positive cells; $2,51-74 \%$ positive cells; $3, \geq 75 \%$ positive cells) and staining intensity ( 0 , negative; 1 , weak; 2 , moderate; 3 , strong). We classified tumors with scores of 0 as having negative expression, 1-2 as having weak positive expression, 3-4 as having moderate expression and 5-6 as having high-level expression.

Cell lines and cell cultures. The EC cell lines Ishikawa3-H-12 (well-differentiated adenocarcinoma) and AN3CA (metastatic undifferentiated EC) were used in this study. The cell lines were kindly provided by Tianjin Medical University, General Hospital. The two cell lines were cultured in DMEM/F12 with $2 \mathrm{mM}$ glutamine and supplemented with $10 \% \mathrm{FBS}$ and $1 \%$ penicillin-streptomycin. Cells were incubated at $37^{\circ} \mathrm{C}$ in a humidified atmosphere of $5 \% \mathrm{CO}_{2}$.

Plasmid transfection and cell transfection. The pcDNA3.1Wnt10b expression vector was purchased from Invitrogen Life Technologies. Full-length WNT10B cDNA was amplified from human placenta RNA by using a primer set (TGGAAGAAT GCGGCTCTGAC and AGAGTGACCTTGGAAGGAAATC). Cell transfections were performed using Lipofectamine 2000 (Invitrogen Life Technologies) according to the manufacturer's instructions. Cells $\left(20 \times 10^{4}\right.$ in $60-\mathrm{mm}$ dish) were transfected with either the Wnt10b expression plasmid (pcWnt10b) or the 
A

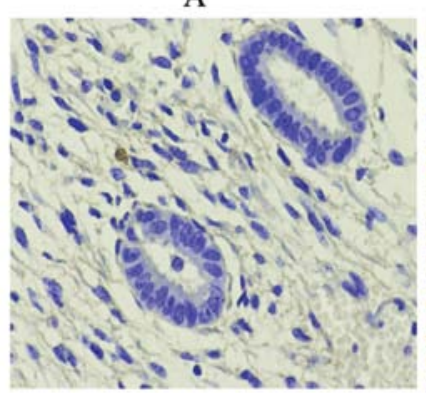

B

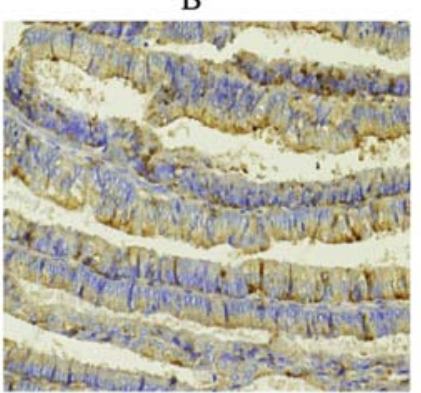

Figure 1. Expression of Wnt10a in normal endometrium and endometrial cancer tissues using immunohistochemistry. (A) Representative results of the negative expression in the proliferative phase endometrium (x400). (B) Representative results of the positive expression in endometrial carcinoma (x400).

empty control vector (pcDNA3.1). The cells were harvested for analysis after $48 \mathrm{~h}$. Western blotting was used to examine the transfection results.

siRNA interference. The target sequence used for WNT10B silencing was AAGGGUGGGAAGGGAUAAU (small interfering siRNA). Cell transfections were performed using Lipofectamine 2000 according to the manufacturer's instructions. Cells $\left(20 \times 10^{4}\right.$ in $60-\mathrm{mm}$ dish) were transfected with $100 \mathrm{nM}$ Wnt10b-specific siRNA or scrambled siRNA and cells were harvested $48 \mathrm{~h}$ after the transfection. Western blotting was used to examine the transfection results.

Protein extraction and western blot analysis. Whole cell extracts were prepared from cultured cells by homogenizing cells in a lysis buffer (10 mM Tris- $\mathrm{HCl}$ (pH 7.5), $150 \mathrm{mM}$ $\mathrm{NaCl}, 1 \% \mathrm{NP}-40)$ containing a cocktail of protease inhibitors. After centrifugation at $15,000 \mathrm{rcf}$ for $30 \mathrm{~min}$ at $4^{\circ} \mathrm{C}$, the supernatants were recovered and used for immunoblot analysis. The proteins were separated by SDS-PAGE and then transferred to polyvinylidene difluoride (PVDF) membranes (Millipore). Blots were blocked and then probed with antibodies against Wnt10b (1:500 dilution), $\beta$-catenin (1:500 dilution), APC (1:500 dilution) and c-myc (1:500 dilution). After washing, the blots were incubated with horseradish peroxidase-conjugated secondary antibodies (1:1,000 dilution) and visualized using Super ECL detection reagent.

MTT cell proliferation assay. A total of $200 \mu \mathrm{l}$ of cells was seeded into a 6 -well microtiter plate at $5 \times 10^{5}$ cells $/ \mathrm{ml}$. We added $10 \mu \mathrm{l}$ of MTT reagent to each well $4 \mathrm{~h}$ before the end of the incubation. After the incubation, the supernatant was removed and $200 \mu 1 \mathrm{DMSO}$ was added to dissolve the formazan crystals. The optical density value (OD) of each sample was measured at a wavelength of $570 \mathrm{~nm}(630 \mathrm{~nm}$ as a reference) on a microplate reader (Multiskan MK3; Thermo Lab Systems). The results of the cell viability measurement were expressed as $\mathrm{OD}_{570}-\mathrm{OD}_{630}$. All experiments were performed in triplicate and the average results were calculated.

Detection of cell apoptosis. Cells were harvested $48 \mathrm{~h}$ posttransfection for apoptosis detection using the Annexin V-FITC

A

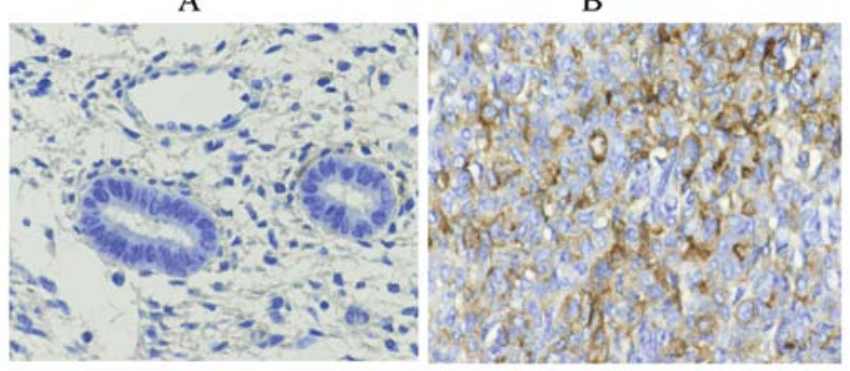

Figure 2. Expression of Wnt10b in normal endometrium and endometrial cancer tissues using immunohistochemistry. (A) Representative results of the negative expression in the proliferative endometrium (x400). (B) Representative results of the positive expression in endometrial carcinoma (x400).

apoptosis detection kit and subsequently analyzed by flow cytometry.

Statistical analysis. Experimental data were analyzed using the SPSS software package (version 17.0) (SPSS, Inc., Chicago, IL, USA). Chi-square and Fisher's exact tests were used to evaluate the significance of differences in categorical variables. Spearman's correlation coefficients were calculated to evaluate the correlation between Wnt10a and Wnt10b expression. Survival rate analyses were compared using Kaplan-Meier survival curves. All P-values were two-sided and a value $<0.05$ was considered to indicate a statistically significant result.

\section{Results}

Expression of Wntloa and Wnt10b proteins in various endometrial tissues. We used IHC to assess Wnt10a and Wnt10b protein levels in human endometrial tissues. We tested the expression of Wnt10a and Wnt10b in EC, simple hyperplasia, complex hyperplasia, atypical hyperplasia endometrium and control normal endometrium groups. The distribution of positive expression of Wnt10a in the proliferative phase, secretory phase, simple hyperplasia, complex hyperplasia, atypical hyperplasia endometrium and endometrial carcinoma cases was $39.58 \%$ (19/48), $44.44 \%$ (16/36), 38.89\% (7/18), $33.33 \%(2 / 6), 30.00 \%(9 / 30)$ and $53.92 \%$ (55/102), respectively $\left(\chi^{2}=2.81, \mathrm{P}=0.25\right)$. There were no significant differences between the positive expression of Wnt10a (Fig. 1). The distribution of positive expression of Wnt10b in the proliferative phase, secretory phase, simple hyperplasia, complex hyperplasia, atypical hyperplasia and endometrial carcinoma cases was $54.17 \%$ (26/48), $27.78 \%$ (10/36), 55.56\% (10/18), 33.33\% $(2 / 6), 60.00 \%(18 / 30)$ and $63.73 \%(65 / 102)$, respectively, and the difference between these groups was significant (Fig. 2) $\left(\chi^{2}=8.12, \mathrm{P}=0.02\right)$. The positive expression of Wnt10b in EC tissues was significantly higher than that in other tissues (Table I and Figs. 1 and 2).

Relationship between Wnt10a and Wnt10b expression and various EC clinicopathological variables. To gain further insight into the clinical significance of Wnt10a and Wnt10b 
Table I. Expression of Wnt10a and Wnt10b in various endometrial tissues.

\begin{tabular}{|c|c|c|c|c|c|c|c|c|c|}
\hline \multirow[b]{2}{*}{ Variables } & \multirow[b]{2}{*}{$\mathrm{N}$} & \multicolumn{4}{|c|}{ Wnt10a } & \multicolumn{4}{|c|}{ Wnt10b } \\
\hline & & $(-)$ & $(+)$ & $(++)$ & $(+++)$ & $(-)$ & $(+)$ & $(++)$ & $(+++)$ \\
\hline \multicolumn{10}{|l|}{ Normal } \\
\hline Proliferative & 48 & 29 & 10 & 6 & 3 & 22 & 11 & 13 & 2 \\
\hline Secretory & 36 & 20 & 10 & 6 & 0 & 26 & 6 & 4 & 0 \\
\hline \multicolumn{10}{|l|}{ Hyperplasia } \\
\hline Simple & 18 & 11 & 2 & 4 & 1 & 8 & 6 & 2 & 2 \\
\hline Complex & 6 & 4 & 2 & 0 & 0 & 4 & 2 & 0 & 0 \\
\hline Atypical & 30 & 21 & 4 & 1 & 4 & 12 & 10 & 5 & 3 \\
\hline Endometrial cancer & 102 & 47 & 32 & 17 & 6 & 37 & 42 & 14 & 9 \\
\hline
\end{tabular}

Table II. Correlation between the expression of Wnt10a and Wnt10b and clinicopathological characteristics in EC cases.

\begin{tabular}{|c|c|c|c|c|c|c|c|c|c|}
\hline \multirow[b]{3}{*}{ Variables } & \multirow[b]{3}{*}{$\mathrm{N}$} & \multicolumn{2}{|c|}{ Wnt10a } & \multirow[b]{3}{*}{$\chi^{2}$} & \multirow[b]{3}{*}{ P-value } & \multicolumn{2}{|c|}{ Wnt10b } & \multirow[b]{3}{*}{$\chi^{2}$} & \multirow[b]{3}{*}{ P-value } \\
\hline & & Negative & Positive & & & Negative & Positive & & \\
\hline & & $\mathrm{n}(\%)$ & $\mathrm{n}(\%)$ & & & $\mathrm{n}(\%)$ & $\mathrm{n}(\%)$ & & \\
\hline Histological type & & & & 4.75 & $\mathbf{0 . 0 3}$ & & & 4.02 & 0.04 \\
\hline Endometrioid & 95 & $41(43.2)$ & $54(56.8)$ & & & $32(33.7)$ & $63(66.3)$ & & \\
\hline Nonendometrioid & 7 & $6(87.5)$ & $1(12.5)$ & & & $5(71.4)$ & $2(28.6)$ & & \\
\hline Grade & & & & 2.06 & 0.36 & & & 6.87 & 0.03 \\
\hline G1 & 42 & 17 (40.5) & $25(59.5)$ & & & $11(26.2)$ & $31(73.8)$ & & \\
\hline G2 & 38 & $17(44.7)$ & $21(55.3)$ & & & $13(34.2)$ & $25(65.8)$ & & \\
\hline G3 & 22 & $13(59.1)$ & $9(40.9)$ & & & $13(59.1)$ & $9(40.9)$ & & \\
\hline Myometrial invasion & & & & 0.10 & 0.75 & & & 2.27 & 0.13 \\
\hline None or $<1 / 2$ & 70 & $33(47.1)$ & $37(52.9)$ & & & $22(28.6)$ & $48(68.5)$ & & \\
\hline$\geq 1 / 2$ & 32 & $14(43.7)$ & $18(56.3)$ & & & $15(51.6)$ & $17(53.1)$ & & \\
\hline FIGO stage & & & & - & 0.54 & & & - & 0.04 \\
\hline I & 75 & $37(49.3)$ & $38(50.7)$ & & & $32(42.7)$ & $43(57.3)$ & & \\
\hline II & 5 & $2\left(2 / 5^{\mathrm{a}}\right)$ & $3\left(3 / 5^{\mathrm{a}}\right)$ & & & $2\left(2 / 5^{a}\right)$ & $3\left(3 / 5^{\mathrm{a}}\right)$ & & \\
\hline III & 22 & $8(36.4)$ & $14(63.6)$ & & & $3(13.6)$ & $19(86.4)$ & & \\
\hline Lymph-node metastasis & & & & - & 0.37 & & & - & 0.02 \\
\hline No & 90 & $40(65.6)$ & $50(55.6)$ & & & $29(32.2)$ & $61(67.8)$ & & \\
\hline Yes & 12 & $7(58.3)$ & $5(41.7)$ & & & $8(66.7)$ & $4(33.3)$ & & \\
\hline
\end{tabular}

${ }^{\mathrm{a}}<10$ samples; no percentage was calculated.

Table III. Correlation between Wnt10a and Wnt10b expression in endometrial cancer tissues.

\begin{tabular}{|c|c|c|c|c|c|}
\hline \multirow[b]{2}{*}{ Wnt10a } & \multirow[b]{2}{*}{$\mathrm{N}$} & \multicolumn{2}{|c|}{ Wnt10b } & \multirow[b]{2}{*}{$\mathrm{r}_{\mathrm{s}}$} & \multirow[b]{2}{*}{$\mathrm{P}$-value } \\
\hline & & - & + & & \\
\hline+ & 55 & 18 & 37 & 0.08 & 0.43 \\
\hline- & 47 & 19 & 28 & & \\
\hline
\end{tabular}

expression in EC, we performed a detailed clinical correlation study. The distribution of Wnt10a and Wnt10b expression among the clinicopathological variables was investigated.
The difference in positive Wnt10a expression in the different histological types was significant. The positive expression of Wnt10a was significantly higher in patients with endometrioid carcinoma $(\mathrm{P}<0.05)$; however, the differences in positive expression between the subgroups for clinicopathological stage, histologic grade, lymph node metastasis and myometrial invasion were not significant $(\mathrm{P}>0.05)$. Positive expression of Wnt10b was evaluated among the histological type, grade and FIGO stage subgroups. The distribution of positive expression of Wnt10b was significantly higher in patients with endometrioid type, high grade, no lymph node metastasis, or advanced stage disease $(\mathrm{P}<0.05)$. There were no significant differences, however, between the myometrial invasion subgroups $(\mathrm{P}>0.05)$ (Table II). 


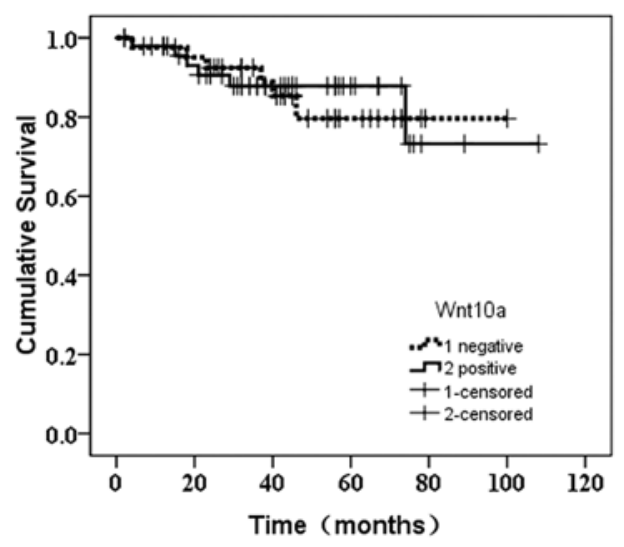

Figure 3. Kaplan-Meier survival curve of EC with or without Wnt10a expression.

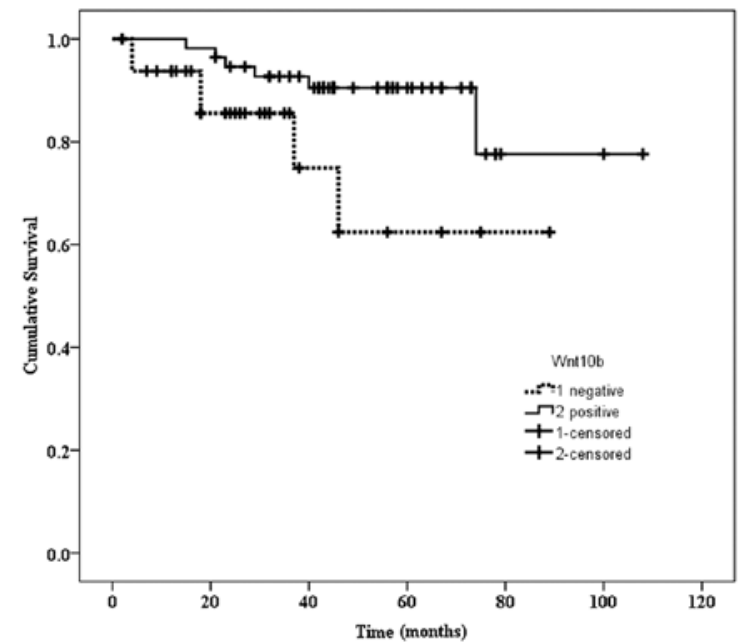

Figure 4. Kaplan-Meier survival curve of EC patients with or without Wnt10b expression.

Correlation of Wnt10a and Wnt10b expression in EC tissues. There was no obvious correlation between Wnt10a and Wnt10b expression in EC ( $\mathrm{P}>0.05)$ (Table III).

Analysis of the prognosis for EC and the expression of Wnt10a and Wnt10b. Twelve of the 102 endometrial carcinoma patients were lost to follow-up. Of the $17(18.9 \%)$ patients who died, $12(70.6 \%)$ died of cancer and $5(29.4 \%)$ died of other causes, including heart failure, renal failure and cerebral hemorrhage. There was no significant difference between the prognosis for patients with and without positive Wnt10a expression $\left(\chi^{2}=0.027, \mathrm{P}=0.868\right)$ (Fig. 3). However, the prognosis for patients with positive expression of Wnt10b was more favorable than that of the negative carriers $\left(\chi^{2}=3.952\right.$, $\mathrm{P}=0.047)$ (Fig. 4).

Impact of Wnt10b expression on EC cell proliferation. To examine the functional role of Wnt10b upregulation in EC, we used the EC Ishikawa3-H-12 and AN3CA cell lines as the in vitro model. The impact of Wnt10b overexpression on cell proliferation was examined via MTT cell proliferation assay. AN3CA cells, in which Wnt10b expression is low, were
A
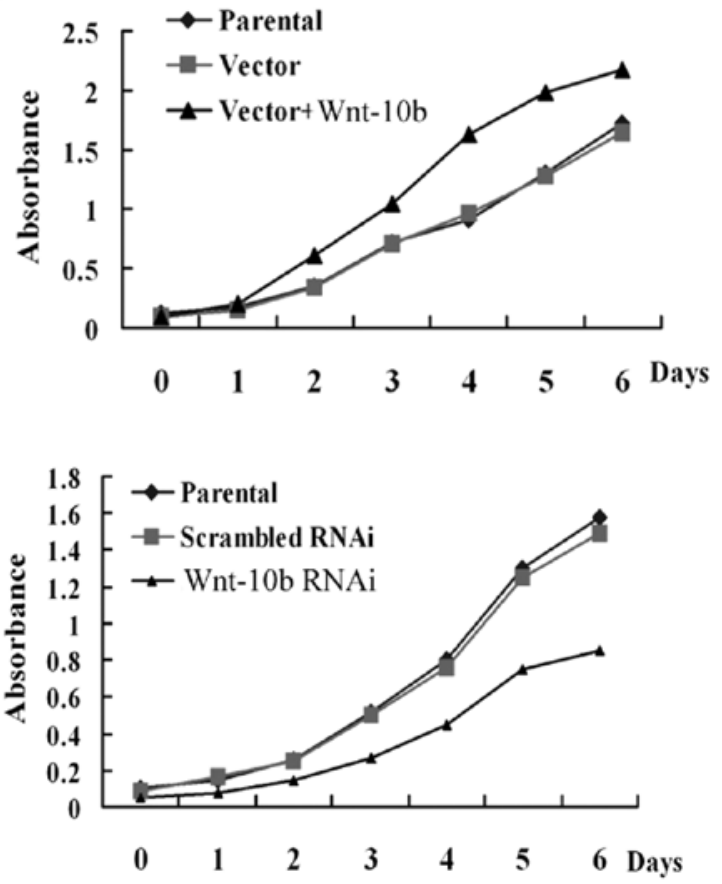

Figure 5. Wnt10b promotes cell proliferation in EC cells, as determined by MTT assay. (A) The proliferation of AN3CA cells with forced Wnt10b expression was higher than that of control groups. (B) The proliferation of Ishikawa3-H-12 cells in the Wnt10b-knockdown group was lower than in the control groups.

transfected with the Wnt10b expression vector ( $\mathrm{pcWnt10b)}$ or empty control vector (pcDNA3.1). The proliferation of cells with forced Wnt10b expression was higher than that of the control groups (Fig. 5A).

To further confirm this result, Ishikawa3-H-12 cells, in which Wnt10b expression is high, were transfected with Wnt10b-specific siRNA to knockdown Wnt10b expression. The results showed that the proliferation of cells in the Wnt10b-knockdown group was lower than that in the control groups (Fig. 5B), showing that Wnt10b expression promotes cell proliferation.

Impact of Wnt10b expression on EC cell apoptosis. The impact of Wnt10b overexpression on AN3CA cell apoptosis was examined via flow cytometry. The percentage of apoptotic cells with forced Wnt10b expression was significantly lower than that of the control groups $(\mathrm{P}<0.05)$ (Fig. 6A and $\mathrm{B})$. Accordingly, the percentage of apoptotic cells in the Wnt10bknockdown group was significantly higher than that in the control group $(\mathrm{P}<0.01)$ (Fig. 7A and $\mathrm{B})$.

Effects of Wntlob expression on $\beta$-catenin, APC and $c$-myc. To determine whether and how the Wnt10b gene plays a role in human EC, we performed a comprehensive analysis of major components of the Wnt signaling pathways in the EC cell lines. We then explored the effects of Wnt10b expression on $\beta$-catenin, APC and c-myc, which are important downstream molecules in the Wnt signal pathway. Both $\beta$-catenin and c-myc expression decreased after Wnt10b knockdown in Ishikawa3-H-12 cells, while APC expression increased (Fig. 8A). Conversely, both $\beta$-catenin and c-myc expression 
A

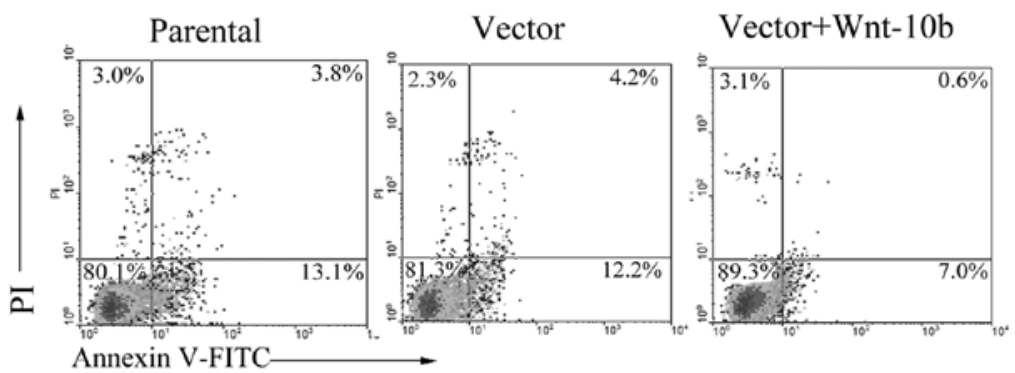

B

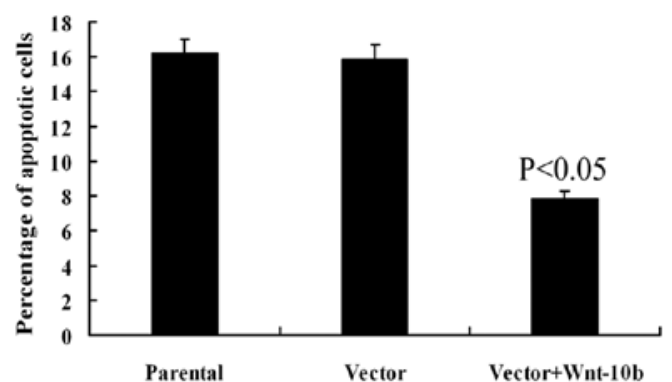

Figure 6. Flow cytometric assay showed that Wnt10b reduces cell apoptosis in AN3CA cells. (A) The percentage of apoptotic cells with forced Wnt10b expression was significantly lower than that of the control groups. (B) The bar chart shows the percentage of apoptotic cells in the three cell groups $(\mathrm{P}<0.05)$.

A
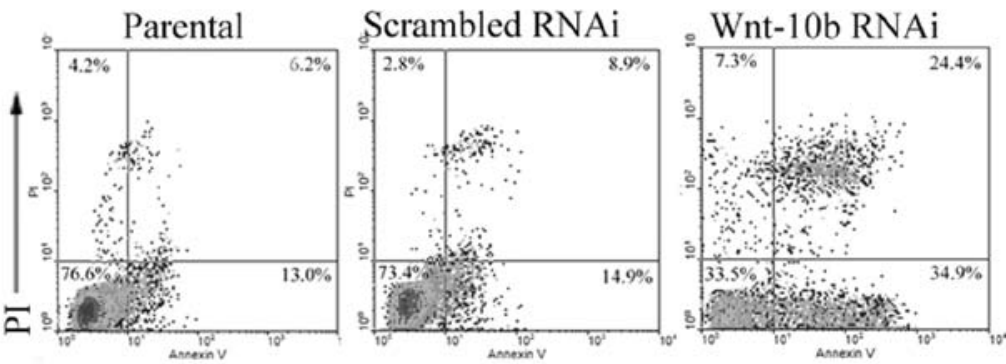

Annexin V-FICT

$\mathrm{B}$

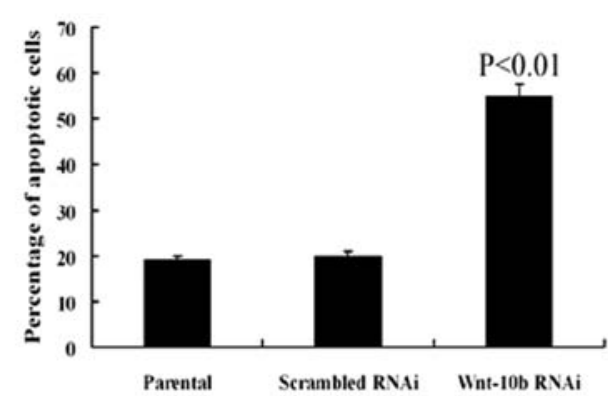

Figure 7. Representative results of the apoptosis assays in Wnt10b-knockdown and control cells at $48 \mathrm{~h}$ using flow cytometry. (A) The percentage of apoptotic cells in Wnt10b-knockdown group was significantly higher than that in the control group. (B) The bar chart shows the percentage of apoptotic cells in the three cell groups $(\mathrm{P}<0.01)$.

increased after Wnt10b-forced expression in the AN3CA cells, while APC expression decreased (Fig. 8B). These results showed that Wnt10b expression had effects on its downstream molecules $\beta$-catenin, APC and c-myc. In our present study, Wnt10b played a critical role in EC; we expected that Wnt10b would activate the $\mathrm{Wnt} / \beta$-catenin pathway in Ishikawa cells. We also observed a correlation between the upregulation of Wnt $10 \mathrm{~b}$ and major components of the Wnt/ $\beta$-catenin pathway. Thus, the role of Wnt10b in the development of EC may occur through the activation of the Wnt/ $\beta$-catenin pathway.

\section{Discussion}

A large number of reports have implicated $\beta$-catenin mutations in 10-45\% of ECs, however, the precise role of Wnt signaling in this disease has not been established (18). Wnt ligands play an important role in regulating the $\mathrm{Wnt} / \beta$-catenin pathway and have therefore been at the forefront of research efforts to investigate the mechanism of Wnt signaling in various solid tumors. Several studies have demonstrated that Wnt protein overexpression is associated with a number of malignant 

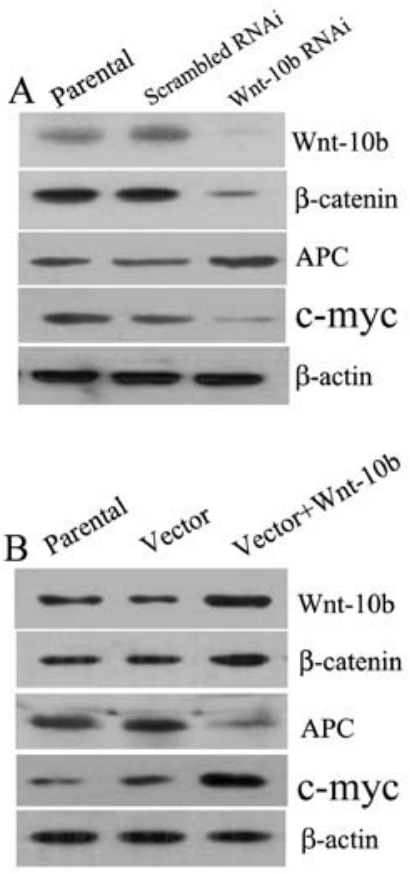

Figure 8. Analysis of the effect of Wnt10b expression on the downstream molecules, $\beta$-catenin, APC and c-myc, of the $\mathrm{Wnt} / \beta$-catenin pathway using western blotting. (A) Wnt10b knockdown in Ishikawa3-H-12 cells decreased both $\beta$-catenin and c-myc expression, but increased APC expression. (B) Conversely, both $\beta$-catenin and c-myc expression increased after forced expression of WNT10b in AN3CA cells, while APC expression decreased.

diseases. The upregulation of Wnt10a may play key roles in certain cases of esophageal, gastric and colorectal cancer (CRC) (19), while the overexpression of Wnt10b is associated with breast cancer (20). Recent interest in the interactions between EC and Wnt signaling has focused on mouse studies involving Wnt10b, a canonical Wnt signaling molecule (21).

Wnt10a overexpression has been reported in esophageal, gastric and CRCs $(19,22)$. Similarly, our present results showed that the positive expression of Wnt10a was higher in EC than hyperplasia or normal endometrium, but the difference was not significant. The positive expression of Wnt10b in cancerous endometrial tissues, however, was significantly higher than hyperplastic or normal endometrium. We also found that the positive expression of Wnt10a in type I endometrial carcinoma was slightly higher than that in type II, which suggests that Wnt10a is likely involved in the estrogen-related carcinogenesis of EC. However, the exact mechanism promoting the development of EC has not yet been elucidated. Our study findings of the upregulation of Wnt10b in both human primary EC tissues and EC cell lines confirm similar reports in breast (23) and prostate carcinomas (24). Wnt10b overexpression has been reported in cancers of the breast and prostate. Importantly, our investigation found that the Wnt10b overexpression was significantly higher in EC than hyperplastic or normal endometria.

A number of clinical pathologies predict the clinical outcome in EC, including stage, grade and histology. Additionally, the depth of myometrial invasion, lymphovascular space invasion (LVSI) and pelvic lymph node status predict the clinical behavior and direct adjuvant treatment options. In our study, we demonstrated that Wnt10b expression is stage-dependent, as Wnt10b expression in advanced stage
EC tissues was reduced. Unfortunately, information about the relationship between the clinicopathological characteristics of EC, Wnt10a and Wnt10b is still not available. Our present findings showed that Wnt10b expression is significantly related to histological type, FIGO stage and lymph node metastasis. The results suggest that higher levels of Wnt10b may contribute to endometrioid, high-grade, advanced-stage and no lymph node metastasis, which suggest favorable prognosis. Regarding the survival curve for Wnt10b, there was a tendency in the current Chinese study towards a better prognosis for EC patients with positive Wnt10b expression than patients who were negative carriers.

We hypothesized that the mechanism of tumor promotion by $\mathrm{Wnt} 10 \mathrm{~b}$ in the Ishikawa cell line is regulated through the Wnt10b-induced upregulation of the Wnt/ $\beta$-catenin pathway. We are the first to show that the EC Ishikawa cell line is responsive to extracellular Wnt signaling. Previous study by our group demonstrated that Wnt10b DNA was amplified in Ishikawa cell lines and not amplified in AN3CA cells (17). In the present study, we showed that the effects of Wnt10b on EC cell proliferation and apoptosis were dependent on the Wnt/ $\beta$-catenin pathway. This result established the Ishikawa cell line as a useful model for the study of Wnt signaling in EC (25). The Ishikawa cell line is a well-differentiated, steroidresponsive EC cell line, with both estrogen and progesterone receptors. The AN3CA cell line is a metastatic, undifferentiated EC line. Benhaj et al (26) found that overexpression of Wnt10b in most breast cancer cell lines determined the molecular mechanisms of Wnt10b-driven mammary tumors via the $\mathrm{Wnt} / \beta$-catenin signaling pathway. Fernandez-Cobo et al (27) also reported that Wnt10b was strongly upregulated in all breast cancer cell lines tested. Their results suggest that tumors express high levels of nuclear $\beta$-catenin. In addition, the expression of typical Wnt target genes, such as c-myc, was strongly activated.

The Wnt/ $\beta$-catenin pathway plays an important role in EC tumorigenesis and development; it activates target genes through the stabilization of $\beta$-catenin in the nucleus. The Wnt genes activate Dishevelled, which recruits $\beta$-catenin and APC to the $\beta$-catenin-TCF (T cell factor) pathway, and TCF stimulates target genes, such as c-myc and cyclin D1, that influence cell proliferation and apoptosis (28). Aberrant activation of Wnt signaling in tumorigenesis has frequently been reported. The prime example is $\mathrm{CRC}$, in which approximately $85 \%$ of cases display a loss-of-function mutation in the tumor-suppressor APC gene (29). In our study, we found that Wnt10b overexpression enhanced the expression of $\beta$-catenin and c-myc and decreased the expression of APC. Wang et al (30) demonstrated that estrogen promotes the proliferation of the endometrium by activating the Wnt/ $\beta$-catenin signaling pathway, which is also a target for progesterone which inhibits the proliferation-promoting effects of estrogen. A number of researchers have suggested that estrogen increases the proliferation of MCF-7 cells by elevating Wnt10b mRNA levels (31). It has been demonstrated that the Wnt family is located upstream of the mammalian target of rapamycin (mTOR) signaling pathway and that increased levels of Wnt10b may promote the activation of the mTOR signaling pathway, resulting in the proliferation of cells. Furthermore, overactivation of mTOR is common in EC tissues and EC cell lines (AN3CA, HEC1A, 
HEC1B, Ishikawa and PL95-2) (32). The development of type I EC has been attributed to the stimulation of unopposed estrogen and this relationship between estrogen and the Wnt signal pathway is likely involved in the carcinogenesis of type I EC. Estrogen enhances the activation of Wnt signaling by promoting the expression of the Wnt protein, resulting in increased levels of c-myc that promote the development of EC. The expression of the Wnt protein promotes endometrial carcinoma by increasing the activation of the mTOR signaling pathway. Moreover, the $\mathrm{Wnt} / \beta$-catenin pathway may also be activated through other mechanisms. The Wnt signaling pathway is a complex signaling network, which is characterized by crosstalk with other altered signaling pathways, such as the Hedgehog and mTOR pathways.

Although our current study provided significant results, there are still some limitations. First, the number of our samples was limited. In the future, large scale research may provide more reliable information. Second, the Wnt $/ \beta$-catenin signaling pathway was involved in the proliferation of EC cells, however, this may not be the only way Wnt is involved in the development of EC. Further research may show us more constructive results.

In summary, the expression level of Wnt10a is higher in endometrioid carcinoma than in non-endometrioid subtypes; however, the underlying mechanism remains unclear. Wnt10b plays an important role in the carcinogenesis of EC, particularly in endometrioid carcinomas. We propose that the expression of Wnt10b is involved in the activation of the Wnt/ $\beta$-catenin pathway, nuclear accumulation of $\beta$-catenin and induction of c-myc overexpression in human EC cells. It has been suggested that Wnt10b is involved in the estrogenpromoting development of EC. As the development of EC is complicated, Wnt10a, Wnt10b and their signaling pathways alone 'cannot tell the entire story'. However, preventive and therapeutic strategies targeting Wnt10a and Wnt10b are likely to be of benefit for EC patients, and further study focused on the relationship among Wnt10a, Wnt10b and EC is warranted.

\section{Acknowledgements}

This study was supported by the Natural Science Fund of China (no. 30772316), the science and technology development fund of Tianjin Municipal Education Commission (no. 20100124) and the foundation of the Fifth Central Hospital of Tianjin (no. 2011208). We also thank Dr Yuanxi Zhu, Wenyan Tian, Huiying Zhang and Dandan Sun for their help in our study.

\section{References}

1. Siegel R, Ward E, Brawley O and Jemal A: Cancer statistics, 2011: The impact of eliminating socioeconomic and racial disparities on premature cancer deaths. CA Cancer J Clin 61: 212-236, 2011

2. Yang D and Han L: Year 1969-2003 study on evolution of endometrial cancer. FuDan Univ J Med Sci 32: 479-483, 2005.

3. Bokhman JV: Two pathogenetic types of endometrial carcinoma. Gynecol Oncol 15: 10-17, 1983.

4. Fleming GF: Systemic chemotherapy for uterine carcinoma: metastatic and adjuvant. J Clin Oncol 25: 2983-2990, 2007.

5. Nusse R: Wnt signaling in disease and in development. Cell Res 15: 28-32, 2005

6. Katoh M: WNT and FGF gene clusters (Review): Int J Oncol 21: 1269-1273, 2002.

7. Wend P, Wend K, Krum SA and Miranda-Carboni GA: The role of WNT10B in physiology and disease. Acta Physiol (Oxf) 204: 34-51, 2012.
8. Modder UI, Oursler MJ, Khosla S and Monroe DG: Wnt10b activates the Wnt, notch and NFKB pathways in U2OS osteosarcoma cells. J Cell Biochem 112: 1392-1402, 2011.

9. White BD, Chien AJ and Dawson DW: Dysregulation of Wnt/ $\beta$ catenin signaling in gastrointestinal cancers. Gastroenterology 142: 219-232, 2012.

10. Robinson DR, Zylstra CR and Williams BO: Wnt signaling and prostate cancer. Curr Drug Targets 9: 571-580, 2008.

11. Mukheriee N, Bhattacharya N, Alam N, et al: Subtype-specific alteration of the Wnt signaling pathway in breast cancer: clinical and prognostic significance. Cancer Sci 103: 210-220, 2012.

12. Gatcliffe TA, Monk BJ, Planutis K and Holcombe RF: Wnt signaling in ovarian tumorigenesis. Int J Gynecol Cancer 18: 954-962, 2008

13. Carmon KS and Loose DS: Development of bioassay for detection of Wnt-binding affinities for individual frizzled receptors. Anal Biochem 401: 288-294, 2010.

14. Kirikoshi H, Sekihara H and Katoh M: WNT10A and WNT6, clustered in human chromosome $2 \mathrm{q} 35$ region with head-to-tail manner, are strongly co-expressed in SW480 cells. Biochem Biophy Res Commun 283: 798-805, 2001.

15. Benhaj K, Akcali KC and Oztuk M: Redundant expression of canonical Wnt ligands in human breast cancer cell lines. Oncol Rep 15: 701-707, 2006.

16. Saegusa M, Hashimura M, Yoshida T and Okayasu I: Beta-catenin mutations and aberrant nuclear expression during endometrial tumorigenesis. Br J Cancer 84: 209-217, 2001.

17. Wang Y, Yang D, Cogdell D, Hu L, Xue F, Broaddus R and Zhang W: Genomic characterization of gene copy-number aberrations in endometrial carcinoma cell lines derived from endometrioid-type endometrial adenocarcinoma. Technol Cancer Res Treat 9: 179-189, 2010.

18. Dellinger TH, Planutis K, Tewari KS and Holcombe RF: Role of canonical Wnt signaling in endometrial carcinogenesis. Expert Rev Anticancer Ther 12: 51-62, 2012.

19. Kirikoshi H, Inoue S, Sekihara $\mathrm{H}$ and Katoh M: Expression of WNT10A in human cancer. Int J Oncol 19: 997-1001, 2001.

20. Brennan KR and Brown AM: Wnt proteins in mammary development and cancer. J Mammary Gland Biol Neoplasia 9: 119-131, 2004.

21. Barbolina MV, Burkhalter RJ and Stack MS: Diverse mechanisms for activation of Wnt signaling in the ovarian tumour microenviroment. Biochem J 437: 1-12, 2011.

22. Kirikoshi H, Sekihara $H$ and Katoh M: Up-regulation of WNT10A by tumor necrosis factor alpha and Helicobacter pylori in gastric cancer. Int J Oncol 19: 533-536, 2001.

23. Milovanovic T, Planutis K, Nquyen A, Marsh JL, Lin F, Hope C and Holcombe RF: Expression of Wnt genes and frizzled 1 and 2 receptors in normal breast epithelium and infiltrating breast carcinoma. Int J Oncol 25: 1337-1342, 2004.

24. Kharaishvili G, Simkova D, Makharoblidze E, et al: Wnt signaling in prostate development and carcinogenesis. Biomed Pap Med Fac Univ Palacky Olomouc Czech Repub 155: 11-18, 2011.

25. Nishida M: The Ishikawa cells from birth to the present. Hum Cell 15: 104-117, 2002.

26. Lustig B and Behrens J: The Wnt signaling pathway and its role in tumor development. J Cancer Res Clin Oncol 129: 199-221, 2003.

27. Fernandez-Cobo M, Zammarchi F, Mandeli J, Holland JF and Pogo BG: Expression of Wnt5A and Wnt10b in non-immortalized breast cancer cells. Oncol Rep 17: 903-907, 2007.

28. Scholten AN, Creutzberg CL, van den Broek LJ, Noordijk EM and Smit VT: Nuclear beta-catenin is a molecular feature of type 1 endometrial carcinoma. J Pathol 201: 460-465, 2003.

29. Polakis P: Wnt signaling and cancer. Genes Dev 14: 1837-1851, 2000.

30. Wang Y, van der Zee, Fodde R and Blok LJ: Wnt/B-catenin and sex hormone signaling in endometrial homeostasis and cancer. Oncotarget 1: 674-684, 2010.

31. Kirikoshi H and Katoh M: Expression and regulation of WNT10B in human cancer: up-regulation of WNT10B in MCF-7 cells by beta-estradiol and down-regulation of WNT10B in NT2 cells by retinoic acid. Int J Mol Med 10: 507-511, 2002.

32. $\mathrm{Lu} \mathrm{Lu} \mathrm{KH,} \mathrm{Wu} \mathrm{W,} \mathrm{Dave} \mathrm{B,} \mathrm{Slomovitz} \mathrm{BM,} \mathrm{Burke} \mathrm{TW,}$ Munsell MF, Broaddus RR and Walker CL: Loss of tuberous sclerosis complex-2 function and activation of mammalian target of rapamycin signaling in endometrial carcinoma. Clin Cancer Res 14: 2543-2550, 2008. 